Gut and Liver, Vol. 9, No. 3, May 2015, pp. 261-262

EDITORIAL

\title{
Practical Suggestions to Train Better-Performing Endosonographers
}

\author{
Eun Young Kim \\ Department of Internal Medicine, Catholic University of Daegu School of Medicine, Daegu, Korea
}

\begin{abstract}
See "Prior Radial-Scanning Endoscopic Ultrasonography Training Did Not Contribute to Subsequent Linear-Array Endoscopic Ultrasonography Study Performance in the Stomach of a Porcine Model" by Wei Xu, et al. on page 353, Vol. 9. No. 3, 2015
\end{abstract}

Endoscopic ultrasonography (EUS) is very useful and frequently employed for diagnostic and therapeutic purposes from many endoscopy units. However, hamper to widespread adoption of EUS is the shortage of experienced endosonographers partly due to the lack of opportunities and insufficiency of formal EUS training programs in the countries in Asia-Pacific region including South Korea. ${ }^{1,2}$

EUS is a highly operator-dependent and technically demanding procedure. EUS requires a good endoscope manipulation technique in conjunction with cognitive ability to catch and interpret ultrasonographic images. Proficiency in EUS requires both adequate training and experiences. The American Society for Gastrointestinal Endoscopy recommended that the minimum number of EUS procedures before assessing competency should include 150 supervised cases (75 being pancreatobiliary), with 50 EUS-guided fine-needle aspiration (EUS-FNA) (at least 25 being pancreatic) in 2001. ${ }^{3}$ Ten years later, Working Group mandated by the British Society of Gastroenterology recommended that EUS trainees should undergo 250 EUS procedures, including 80 luminal cancers, 20 subepithelial lesions, and 150 pancreatobiliary cases (at least half of which are likely pancreatic adenocarcinoma). A total of at least 75 FNA should be performed, of which at least 45 are likely pancreatic adenocarcinomas. $^{4}$

The learning curve of EUS is relatively lengthy. To avoid complications and assure examination quality, high standard of training is necessary. There are many ways to learn EUS other than real supervised practice on actual patient. Lectures and watching video clips can be helpful in gaining basic knowledge. Direct observations of proficient endosonographers' perfor- mances can provide important tips. Computer-based simulators, phantoms, ex vivo models, and live animal models are also applicable. Computer-based simulators such as EUS Mentor ${ }^{\circledR}$ (Simbionix, Tel Hashomer, Israel) has been developed. ${ }^{5}$ EUS Mentor is a computer-based endoscopic simulator platform which allows trainees to gain experience in manipulating the scope and wheels and it also provides realistic radial and linear-array EUS images based on human anatomy. EUS Meets Voxel-man ${ }^{\circledR}$ is an interactive anatomic simulation program which gives threedimensional anatomic animation of linear-array endoscopic ultrasound images. ${ }^{6}$ Phantoms are also introduced. The Ikuma model was designed through collaboration between Olympus Medical Systems Corporation (Japan) and Kyoto Kagaku (Japan) to simulate real-life human anatomy. Trainee can practice navigational skill with this model but tactile feeling is far from that of real patient. Ex vivo animal model such as EUS RK model gives enhanced realistic feeling and EUS-FNA is feasible with normal EUS equipment. ${ }^{7}$ However, this model does not provide simulated blood flow. Live animal models are the most realistic simulator offering the best training experience. Porcine models resemble human anatomy and human-like EUS images can be obtained from transgastric scanning. EUS training in live animal model significantly improves performance, confidence, and procedural comfort of trainee when returning to real patient examinations. $^{8}$

There are two kinds of echoendoscope. Radial or sector echoendoscope (RS) provides 360 degree circumferential view similar to computed tomography images. Linear or convex or curved linear-array echoendoscope (CLA) has limited field of ultrasonic view, less than 180 degree, and orientation is more difficult for

Correspondence to: Eun Young Kim

Department of Internal Medicine, Catholic University of Daegu School of Medicine, 33 Duryugongwon-ro 17-gil, Nam-gu, Daegu 705-718, Korea

Tel: +82-53-650-4092, Fax: +82-53-624-3281, E-mail: kimey@cu.ac.kr

pISSN 1976-2283 eISSN 2005-1212 http://dx.doi.org/10.5009/gnl15124

@) This is an Open Access article distributed under the terms of the Creative Commons Attribution Non-Commercial License (http://creativecommons.org/licenses/by-nc/3.0) which permits unrestricted non-commercial use, distribution, and reproduction in any medium, provided the original work is properly cited. 
the beginners. However, trainee does not need to learn one by one and both can be integrated from the basic EUS training course. In this issue of Gut and Liver, Xu et al. ${ }^{9}$ report that previous RS training did not help on subsequent CLA performance in the stomach of porcine model. They randomly allocated 18 freshman trainees into two groups, A and B. Group A received a 15-minute hands-on practice for RS first and then a 15-minute hands-on practice was done for CLA with group B who did not received RS training in advance. They found that the vast majority of trainees enhanced their EUS skills greatly including interventional procedures regardless of whether they received 15-minute RS training in advance or not. Fifteen-minute training with porcine model can improve the EUS skills of trainee but it seems that is not enough to grow their competency for real task. In addition, transgastric examination is very limited and easier than transduodenal scanning, while more difficult transduodenal approach is not usually performed due to the anatomical uniqueness of porcine model.

Recently, many EUS centers focus on interventional EUS procedures which are only possible with CLA. Delineation capability of CLA is comparable with RS for most targets and better for portal area and bifurcation area of major vessels. ${ }^{10}$ That is why when a center is limited to have only one scope, CLA scope is preferred. Some centers use only CLA even though they have both scopes to save time and trainees learn only CLA. Nevertheless, RS has its own advantages as a diagnostic tool and both procedures are complementary. In that aspect, training for both procedures should be mandatory for novice trainees.

Field of EUS has evolved remarkably during the past few decades. EUS is a technically challenging procedure and learning EUS is time-consuming and tedious. Well-structured training program including live animal model will help both mentors and trainees to shorten the training time.

\section{CONFLICTS OF INTEREST}

No potential conflict of interest relevant to this article was reported.

\section{REFERENCES}

1. Lee TH, Kim EY, Kim J0, Lee KH, Lee JS; Korean EUS Study Group. South Korean endoscopists' attitudes toward endoscopic ultrasound for the evaluation of gastrointestinal diseases. Turk $\mathrm{J}$ Gastroenterol 2014;25:63-69.

2. Ho KY. Survey of endoscopic ultrasonographic practice and training in the Asia-Pacific region. J Gastroenterol Hepatol 2006;21: 1231-1235.

3. Eisen GM, Dominitz JA, Faigel D0, et al. Guidelines for credentialing and granting privileges for endoscopic ultrasound. Gastrointest Endosc 2001;54:811-814.

4. Meenan J, Harris K, Oppong K, et al. Service provision and training for endoscopic ultrasound in the UK. Frontline Gastroenterol 2011;2:188-194.

5. Cantù P, Penagini R. Computer simulators: the present and near future of training in digestive endoscopy. Dig Liver Dis 2012;44 106-110.

6. Burmester E, Leineweber T, Hacker S, Tiede U, Hütteroth TH, Höhne KH. EUS Meets Voxel-Man: three-dimensional anatomic animation of linear-array endoscopic ultrasound images. Endoscopy 2004;36:726-730.

7. Matsuda K, Tajiri H, Hawes RH. How shall we experience EUS and EUS-FNA before the first procedure? The development of learning tools. Dig Endosc 2004;16(Suppl 2):S236S239.

8. Fritscher-Ravens A, Cuming T, Dhar S, et al. Endoscopic ultrasound-guided fine needle aspiration training: evaluation of a new porcine lymphadenopathy model for in vivo hands-on teaching and training, and review of the literature. Endoscopy 2013;45:114120.

9. Xu W, Liu Y, Pan P, Guo Y, Wu RP, Yao YZ. Prior radial-scanning endoscopic ultrasonography training did not contribute to subsequent linear-array endoscopic ultrasonography study performance in the stomach of a porcine model. Gut Liver 2015;9:353-357.

10. Kaneko M, Katanuma A, Maguchi H, et al. Prospective, randomized, comparative study of delineation capability of radial scanning and curved linear array endoscopic ultrasound for the pancreaticobiliary region. Endosc Int Open 2014;02:E160-E170. 